\title{
Corrigendum
}

\section{Corrigendum to "Study QoS Optimization and Energy Saving Techniques in Cloud, Fog, Edge, and IoT"}

\author{
Zhiguo Qu, Yilin Wang, Le Sun (D), Dandan Peng, and Zheng Li \\ Jiangsu Collaborative Innovation Center of Atmospheric Environment and Equipment Technology (CICAEET), \\ Nanjing University of Information Science \& Technology, 210044 Nanjing, China \\ Correspondence should be addressed to Le Sun; sunle2009@gmail.com
}

Received 16 May 2020; Accepted 16 May 2020; Published 9 July 2020

Copyright ( 2020 Zhiguo Qu et al. This is an open access article distributed under the Creative Commons Attribution License, which permits unrestricted use, distribution, and reproduction in any medium, provided the original work is properly cited.

In the article titled "Study QoS Optimization and Energy Saving Techniques in Cloud, Fog, Edge, and IoT" [1], the corresponding author requested to remove the first affiliation, "Engineering Research Center of Digital Forensics, Ministry of Education, Nanjing, China" for all the authors. The corrected author affiliations are shown above.

\section{References}

[1] Z. Qu, Y. Wang, L. Sun, D. Peng, and Z. Li, "Study QoS optimization and energy saving techniques in cloud, fog, edge and IoT," Complexity, vol. 2020, Article ID 8964165, 16 pages, 2020. 